\title{
Fabrication of silver doped nano hydroxyapatite-carrageenan hydrogels for articular cartilage applications
}

\author{
Subhasmita Swain ${ }^{1}$, Tae Yub Kwon², Tapash R. Rautray ${ }^{1^{*}}$ \\ ${ }^{1}$ Biomaterials and Tissue Regeneration Laboratory, Centre of Excellence in Theoretical and \\ Mathematical Sciences, Siksha 'O' Anusandhan (Deemed to be University), Khandagiri Square, \\ Bhubaneswar - 751030, Odisha, India \\ ${ }^{2}$ Department of Dental Biomaterials, School of Dentistry, Kyungpook National University, \\ Samduk-dong, Jung-gu, Daegu, India
}

\begin{abstract}
:
It can be found from the results that nano hydroxyapatite- silver $-3.0 \mathrm{wt} \%$ carageenan (nHA-AgCG3.0) improved the mechanical properties of the as-formed hydrogel scaffold after incorporation of higher CG concentration. The Young's modulus of hydroxyapatite- silver $1.5 \mathrm{wt} \%$ carageenan (nHA-Ag-CG1.5) was found to be $0.36 \pm 0.07 \mathrm{MPa}$ that increased in case of nHA-Ag-CG3.0 demonstrating better interfacial compatibility of their matrix with respect to the reinforcement. This increase in reinforcement concentration resulted in higher stiffness that dissipated energy. The higher swelling ratio is envisaged to induce better cell adhesion and proliferation. The biodegradability test was performed in phosphate buffered saline at body temperature for 3 weeks. The biodegradability rate of nHA-Ag-CG1.5 was found to be equivalent to nHA-Ag-CG3.0 hydrogels at day 7 while it increased faster in nHA-Ag-CG3.0 on days 14 and 21 that may be ascribed to the possible interaction of nHA and Ag with their CG matrix. The bacterial cell viability of Staphylococcus aureus (S. aureus) was performed after 10 $\mathrm{h}, 20 \mathrm{~h}$ and $30 \mathrm{~h}$ of culture. The nHA-Ag-CG1.5 exhibited restrained growth of S. aureus as compared to nHA-Ag-CG3.0 and these results were validated by CLSM analysis. Hence, nHAAg-CG3.0 may be considered to have more cytocompatibility than nHA-Ag-CG 1.5.
\end{abstract}

Keywords: Hydrogels; hydroxyapatite; carageenan; silver.

\section{Introduction}

The replacement of bone tissues caused due to accident or disease has become a new challenge in the field of biomaterials science. Therefore, fabricating a novel biomaterial having outstanding bone compatible properties that would replace the currently used biomaterials with many drawbacks has become vital in the field of tissue engineering. With the use of either physical or

${ }^{*}$ Corresponding author, Email: tapash.rautray@ gmail.com, Tel.: +91-9040000604 
chemical crosslinking, a network of 3D hydrophilic structure termed as scaffolds, can be fabricated which have promising physical and biological properties including high water retention, favorable biocompatibility and lowest inflammation capability equivalent to natural functional tissues [1-4].

Production of scaffolds from biocompatible materials has recently been getting serious attention in biomedical engineering since they offer bio-physico-chemical pathways of increasing and restoring natural tissues. A 3D framework that mainly operates as a supporting structure for body cells to adhere and proliferate thus permitting them to grow and achieve their functions [5, 6]. As soon as the cells start to experience proliferation, degradation of the scaffold initiate and are slowly absorbed by the body. Gradually the scaffold gives space to the cells for more regeneration and the new tissues formed organize to take the total shape of scaffold. So as to facilitate regeneration of new tissues, some biomimetic and structural requirements are taken into account. A biomedically ideal scaffold must be biocompatible having interconnected pore channels so as to allow cell migration, supply oxygen and nutrients. The scaffold would promote cell adhesion, proliferation, differentiation and have controlled biodegradable rate having adequate mechanical strength to act as the support system for the cells [7-9].

HA is the primary inorganic constituent of bone and teeth that can be used as a potential reinforcement in hydrogels for bone tissue engineering applications. HA has long been widely used as bone substitute materials. In fact, because of its high osteoconductivity, excellent biocompatibility and bioactivity, HA can be extensively used as a guided bone regeneration material in spite of its low degradation rate and fragility [10-12].

Because of its slow degradation rate, application of HA as bioresorbable material is limited. To overcome this drawback, other calcium phosphate materials with higher solubility have been investigated to be used as bone-substitutes. Additional components such as $\mathrm{Sr}, \mathrm{Mg}, \mathrm{Ag}, \mathrm{Cu}, \mathrm{Zn}$ etc. are added to them for improving and inducing new properties [13, 14].

CG is a derivative of a marine algae called 'Rhodophyceae' and is a high molecular weight anionic heteropolysaccharide. It is a low cost, non-toxic, biocompatible and hydrophilic marine based linear polysaccharide. Moreover, the sulfate groups present in it has the potential to mimick glycosaminoglycans (GAGs) which are the negatively charged macromolecules [15, 16]. $\mathrm{CG}$ in combination with HA would be a potential candidate in orthopaedic applications.

Furthermore, the gel strength has been found to be higher in case of less sulfate concentration in carrageenan and the least ester sulfate is available in $\kappa$-carrageenan that can be most appropriate for applications in bone scaffolds. In addition, GAGs which are the constituent of bone and 
cartilage of humans have been shown to have structurally analogous to $\kappa$-carrageenan ( $\kappa-\mathrm{CG})$ [17].

The properties of k-carrageenan incorporated HA implants are improved as compared to only HA based implants. Hence, k-carrageenan incorporated HA is likely to achieve the requirements of bioresorbability and biocompatibility for use as scaffolds. However, less number of applications of k-carrageenan have been available that includes micro-encapsulation along with immobilization of certain drugs and wound dressing [18].

Infection has posed to be a major threat in orthopedic implants leading to revision surgery or implant failure. Hence it is a challenging job of treating these infections that may lead to serious complications resulting in amputation and mortality. Infections at the implant sites results in biofilm formation. Because of the higher demand for novel biocompatible materials having antibacterial properties, the researchers started to incorporate antibacterial materials in biomaterials [13, 19]. Ag has got significant importance as an antibacterial agent and has extensively been used in various biomedical applications.

To mimick the natural bone structure, a novel promising approach has been taken in the present study for the fabrication of hydrogels to regenerate the bone and cartilage defect sites simultaneously providing antibacterial efficacy. The sol-gel based methodology was employed to fabricate and maintain the nano-HA precipitates while a lyophilization technique was used to print the structure of the hydrogel.

\section{Materials and methods}

\subsection{Specimen preparation}

nHA was prepared by taking a $\mathrm{Ca} / \mathrm{P}$ ratio of 1.67 by dropwise addition of $0.6 \mathrm{M}$ calcium hydroxide with $0.4 \mathrm{M}$ orthophosphoric acid and adjusting its $\mathrm{pH}$ to 12 . The solution was stirred vigorously at $1500 \mathrm{rpm}$ for $12 \mathrm{~h}$ and were drop wise added to k-carrageenan solution under stirring $\left(10{ }^{\circ} \mathrm{C}, 20 \mathrm{~min}\right)$ condition. $0.7 \% \mathrm{Ag}\left(\mathrm{NO}_{3}\right)_{2}$ solution was dropwise added to the above solution. Two separate specimens were prepared with CG concentration of $1.5 \mathrm{wt} \%$ and $3 \mathrm{wt} \%$. To obtain homogeneity, samples were stirred at $90 \mathrm{rpm}\left(40^{\circ} \mathrm{C}, 24 \mathrm{~h}\right)$ and ultrasonicated for $2 \mathrm{~h}$. The nHA-Ag-CG gels were decanted into Teflon molds quenched to $-80^{\circ} \mathrm{C}$ and lyophilized for $36 \mathrm{~h}$. 


\subsection{Swelling studies}

The water-absorption capability of the prepared specimens was examined as per equation (1). For measuring the water absorption capability, a known quantity of the lyophilized hydrogel $(W d)$ was immersed in a phosphate buffered saline (PBS) solution at $\mathrm{pH} 7.4$ and $37^{\circ} \mathrm{C}$. The wet hydrogel was taken out of PBS and after wiping off the excessive adsorbed surface PBS, it was weighed to find out the weight during swelling $(W s)$ at different durations (20- and 40- min, 1-, 2-, 4-, 8-, 24-, 48-, and 72-hours). The swelling ratio was measured using the equation

$$
\text { Swelling } \%=\frac{W s-W d}{W d} \times 100
$$

\subsection{Degradation study}

The degradation profile of the prepared specimens was examined as per equation (2). The initial weight $(W i)$ of the lyophilised hydrogels were noted and they were soaked in PBS of $\mathrm{pH}$ 7.4. The incubation of hydrogels was carried out at $37^{\circ} \mathrm{C}$ and placed in a shaker at $80 \mathrm{rpm}$. The PBS was renewed every three days. At time points of 3-, 7-, 14-, 21-, and 28- days, the hydrogels were rinsed with water and lyophilized. The dried hydrogels were then weighed $(W f)$ and the degradation profile was measured as per the following equation (2):

Degradation $\%=\frac{W i-W f}{W i} \times 100$

The data presented are the mean \pm standard deviation (SD) of five measurements for each hydrogel in swelling and degradation behaviour.

\subsection{Contact angle measurement}

The water wetting angle or hydrophilicity of the hydrogel was quantified by measuring their angle subtended with a drop of PBS at $\mathrm{pH}$ 7.4. The contact angle was measured using a Contact angle detection system (Kruss, Germany). The data represented are the mean \pm SD of five measurements for each hydrogel [12].

\subsection{Mechanical properties}

Compressive tests were used to study the mechanical properties through two modes such as (i) the hydrogels were compressed till its breaking point and (ii) the hydrogels were placed on cyclic compression test. Initial test was employed to measure the Young's modulus and compressive 
strengths while the degradation behavior of hydrogels was estimated from the second test. Hydrogel blocks ( $8 \mathrm{~mm}$ diameter x $5 \mathrm{~mm}$ thickness, disk shape) were used in both the tests. The solutions of hydrogels were transferred to a $8 \mathrm{~mm}$ x $5 \mathrm{~mm}$ circular pit and irradiated with UV for photocrosslinking. The hydrogel specimens were then dipped in PBS solution for $10 \mathrm{~h}$. The hydrogels in swelling condition were subjected to compression until break using a universal testing machine (UNITEST M2, Test One Co. Ltd., Korea) (load: 100 N, Strain: 5 mm/min, 25 ${ }^{\circ} \mathrm{C}$ ) in order to measure their compressive strength and Young's modulus. Moreover, the durability test was carried out by cyclic compression test as performed earlier. $60 \%$ applied load was given for compression and data collected were from 50 cycles. The data were the mean of three tests with SD.

\subsection{Bacterial viability test}

S. aureus was taken for the antibacterial efficacy of the specimens. The strain of bacteria was cultured in MH Broth medium [20]. MTT assay was taken to quantify the in vitro bacteria viability. The specimens were placed in a 24-multiwell plate each carrying $1 \mathrm{~mL}$ bacteria suspension with an amount of $1 \times 10^{6} \mathrm{CFU} / \mathrm{mL}$ and incubated at $37{ }^{\circ} \mathrm{C}$ in a $\mathrm{CO}_{2}$ atmosphere. The in vitro culture of bacteria was performed for 10,20 and $30 \mathrm{~h}$ and the bacteria adhered to the substrate were quantified for bacterial viability by inoculation of equal quantities $(200 \mu \mathrm{L})$ of bacterial suspension and MTT solution $(5 \mathrm{mg} / \mathrm{mL})$ and they were then placed for incubation at $37{ }^{\circ} \mathrm{C}$ for $10 \mathrm{~h}$ until the formation of formazan crystals. The OD of the specimen was calculated using a spectrophotometric microplate reader at $490 \mathrm{~nm}$ [19].

\subsection{In Vitro Cell Culture}

Human osteoblast like cells (MG63) were cultured in DMEM added with $10 \%$ fetal calf serum (FCS, HiMedia) and incubated in $5 \% \mathrm{CO}_{2}$ at $37{ }^{\circ} \mathrm{C}$. The culture media was renewed every alternate day. After achieving confluence, the non-viable cells were removed by a trypsin-EDTA solution $(0.5 \mathrm{~g} / \mathrm{L}$ trypsin and $0.2 \mathrm{~g} / \mathrm{L}$ EDTA, Gibco) and the cells were shifted to a new tissue culture flask [21].

\subsection{Cell Counting Kit-8 (CCK-8) Assay}

CCK-8 assay (Dojindo laboratories, Kumamoto, Japan) was used to assess the MG63 cell proliferation of hydrogels. The specimens were placed in a 96-multiwell plate. 1 x $10^{4}$ cells/well 
were added on each well and incubated for 1, 7 and 14 days. The specimens were washed thrice with PBS solution to remove the non-viable cells. The specimens were added with $10 \mu \mathrm{L}$ of CCK-8 solution and incubated for $3 \mathrm{~h}$. The optical density (OD) was then quantified with the help of a microplate reader at $490 \mathrm{~nm}$. For each incubation time of 1, 7 and 14 days, the absorbance values were taken as the mean of three measurements [21].

\subsection{Osteogenic expressions}

The osteoblastic expression of genes such as RUNX2, collagen type I (COL1) and osteocalcin (OC) were assessed using RT-PCR. RNA, extracted with Rneasy® Mini Kit (QIAGEN) as per Instruction manual was measured using UV-VIS spectrophotometry at $260 \mathrm{~nm} .0 .5 \mu \mathrm{g}$ of RNA was reverse transcribed and amplified ( 25 cycles) with RT-PCR at $55{ }^{\circ} \mathrm{C}$. Table 1 depicts the primers used in RT-PCR analyses. ImageJ 1.41 software was used to analyse the bands obtained from the electrophoresis of a $1 \%(\mathrm{w} / \mathrm{V})$ agarose gel [21].

\subsection{Confocal laser scanning microscopy (CLSM) analysis}

After incubating the specimens for a week, the osteoblast cells were fixed in (4\% paraformaldehyde) and then permeabilized by $0.5 \%$ Triton X-100. The DAPI (4',6-diamidino-2phenylindole, Sigma-Aldrich) fluroscence staining was employed for visualizing nuclei. For observation of osteoblast morphology, images were subjected to CLSM (Leica TCS SP8, Wetzlar, Germany) analysis [19].

\section{Statistical analysis}

The experimental values were reproduced as mean \pm standard deviation (SD) and the difference in values of data were reciprocated by one way analysis of variance (ANOVA).

\section{Results and discussion}

Tissue engineering has played a vital role in overcoming major trauma and damaged tissues or organs. Hydrogels have been found to be emerging scaffolds with regenerating impacts on cartilage, soft and hard tissues. Injectable hydrogels have self-healing characteristics that permits the gel to obtain desirable mechanical strength by tissue in-growth [22]. Gelling of hydrogels at $\mathrm{pH} 7.4$ and $37{ }^{\circ} \mathrm{C}$ are the emerging biomaterials where gelation occurs due to body temperature 
without the help of any chemical or mechanical stimuli. Furthermore, these hydrogels can be directly applied in a minimally invasive method [23].

It has long been established that HA and carageenan have regenerative properties as far as vascularization in human bones are concerned. However, because of their higher biocompatible properties they are prone to bacterial infection [24]. Hence, simultaneous presence of an antibacterial agent such as Ag in a hydrogel matrix would provide the dual action of hydrogel in terms of biocompatibility and antibacterial efficacy.

\subsection{Swelling ratio determination}

Swelling is a vital property of hydrogels that stimulates the gas exchange, helps in absorbing body fluid, transferring nutrients, encapsulating and growing cells within the hydrogel. Fig. 1 depicts the swelling ability of hydrogel.

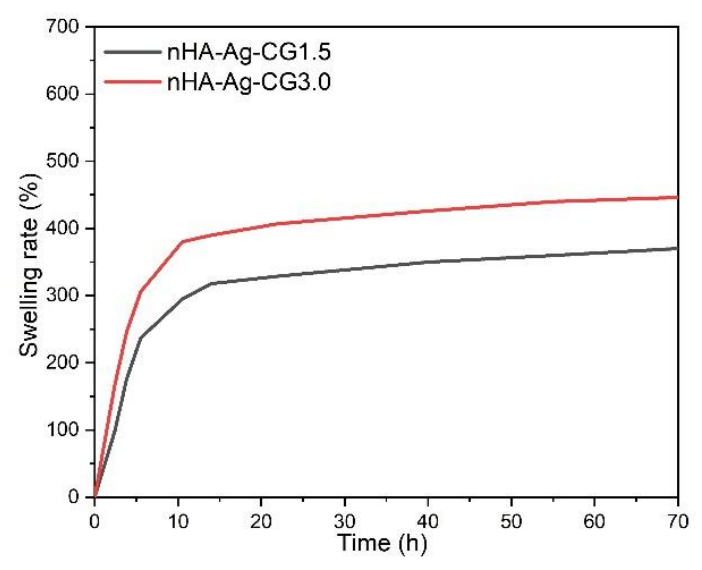

Fig. 1. Swelling ability

While nHA-Ag-CG3.0 exhibited higher swelling ratio (500\%), it was significantly reduced (400\%) in nHA-Ag-CG1.5 hydrogels. The higher swelling ratio is envisaged to induce better cell adhesion and proliferation. Greater swelling index helps in transport of nutrients and fluid uptake from the media near its vicinity. Enhanced swelling ratio may also be due to higher hydrophilicity of carageenan [25]. The swelling equilibrium was attained at $7 \mathrm{~h}$ for $\mathrm{nHA}-\mathrm{Ag}$ CG3.0 while nHA-Ag-CG1.5 attained it at $3 \mathrm{~h}$. From these results, it can be inferred that the incorporation of higher CG concentration in nHA-Ag-CG1.5 hydrogel decreased the swelling ratio. Absorption of body fluid through swelling of the hydrogels is the basic necessity in tissue engineering constructs. In the functions of hydrogels, swelling and water retention ability are primarily related to the amorphous regions and free $\mathrm{OH}^{-}$radicals in the polymer. The swelling rate of the hydrogels should be able to efficiently absorb the biological fluids that come in 
contact with them. Hence, the evaluation of swelling performance of the hydrogels is a vital criterion.

\subsection{Biodegradability evaluation}

Biodegradability is another vital characteristic of the hydrogels such that once the functional site of the hydrogels is completely regenerated; the scaffold on the other hand has to degrade concurrently. The biodegradability test was performed in PBS at body temperature for 3 weeks. The biodegradation rate of nHA-Ag-CG1.5 was found to be equivalent to nHA-Ag-CG3.0 hydrogels at day 7 while the biodegradation rate increased faster in nHA-Ag-CG3.0 on days 14 and 21 that may be attributed to the possible interaction of nHA and Ag with their CG matrix. The results from swelling and degradation behavior were found to be interrelated. Higher swellable matrices interact with more number of $\mathrm{H}_{2} \mathrm{O}$ molecules that result in quicker degradation of the hydrogels and slowly degrading matrix showed lower swelling ratio [26]. Hence both the results depicted that higher swelling and faster degradation of nHA-Ag-CG3.0 was a consequence of higher CG concentration.

\subsection{Contact angle measurements}

Water wetting angle of the surface of a biomaterial plays a vital role that determines the adhesion of proteins or osteoblast cells on its surface [12]. The hydrophilicity of the hydrogel was obtained by measuring the angle between the hydrogel surface with that of simulated body fluid. It was found from the study that the as-formed hydrogel was hydrophilic in nature with a contact angle of $32^{\circ}$. The water wetting ability of a biomaterial surface largely impacts the cell adhesion, proliferation and differentiation based on their hydrophilicity [27].

\subsection{Evaluation of mechanical properties}

Incorporation of metallic antibacterial agents into polymeric blends at physiological temperature and $\mathrm{pH}$ has stiffened the hydrogels. Hydrogels having higher (3\%) concentrations of $\mathrm{CG}$ showed to have higher tensile strength than the lower (1.5\%) concentration of CG. Higher CG concentration also provided higher stiffness (20.67 MPa) than its lower CG (18.79 $\mathrm{MPa})$ counterpart [26, 28]. In the composite hydrogels, increased levels of compressive strength is obtained as compared to hydrogels without inorganic components which is attributed to the 
reinforcement that develops secondary cross linking points arresting free movement of polymer chain thus making the hydrogels stiff.

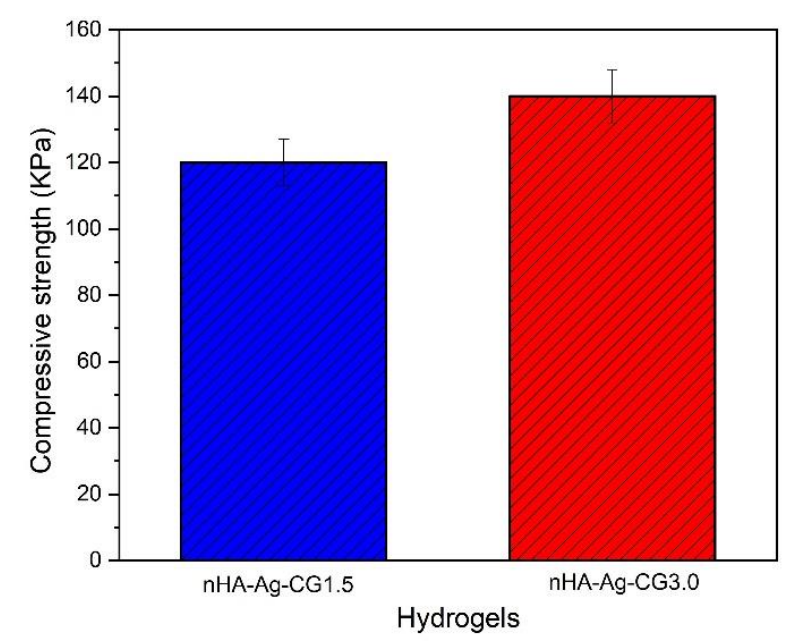

Fig. 2. Compressive strengths of the specimens

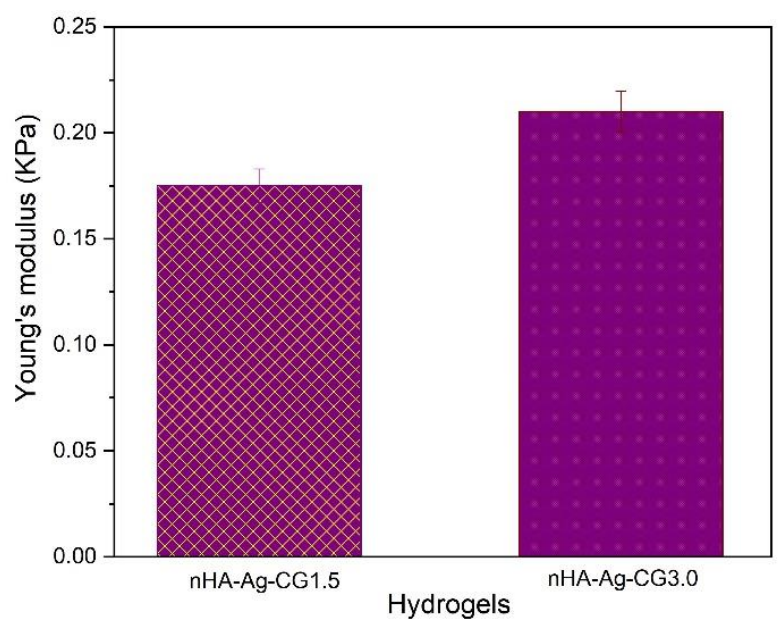

Fig. 3. Young's modulus of the specimens

The hydrogel scaffolds having adequate load bearing capacity have shown to be ideal as biomedical material. Hence, the test to evaluate the compressive strength for both the hydrogels were performed. Howsoever, the compressive strength was in the order of nHA-Ag-CG3.0 (11.2 $\mathrm{MPa})>$ nHA-Ag-CG1.5 (9.1MPa). It can be found from Fig. 2 and Fig. 3 that the compressive strength and Young's modulus were considerably higher in nHA-Ag-CG3.0 than nHA-AgCG1.5. The Young's modulus of nHA-Ag-CG1.5 is $0.36 \pm 0.07 \mathrm{MPa}$ that increased in case of nHA-Ag-CG3.0 (0.54 $\pm 0.09 \mathrm{MPa})$ demonstrating better interfacial compatibility of their matrix with respect to the reinforcement. This increase in reinforcement concentration results in higher stiffness that dissipate energy. Hence, it can be found from the results that nHA-Ag-CG3.0 improved its mechanical properties after incorporation of higher CG concentration. As the hydrogels are stressed, the entangled chains of polymeric matrix receive the load and gets reoriented resulting in draining out of interstitial fluid. Scaffold structure with adequate mechanical strength plays an important role for a successful implant application. Higher mechanical strength in both the hydrogels may be attributed to the homogeneous distribution of hydroxyapatite particles.

\subsection{Antibacterial activity}

In order to assess the antibacterial efficacy of $\mathrm{Ag}$ present in the hydrogels, their antibacterial activities were evaluated using S. aureus pathogenic bacteria. While the concentration of Ag was same in both the hydrogels, the antibacterial activities of both of the hydrogels were expected to 
be equivalent. Hence, a comparison of the antibacterial activities of nHA-Ag-CG3.0 hydrogel with that of nHA-Ag-CG1.5 hydrogels is depicted in Fig. 4. Antibacterial Ag blocked the channels of bacteria cells and the positive charge of $\mathrm{Ag}$ neutralize the negatively charged bacteria membrane changing the lipid layer structure, increasing the permeability of cell membranes and ruptured the bacterial membrane resulting the bacteria cell death [13, 29]. The bacterial cell viability of $S$. aureus was performed after $10 \mathrm{~h}, 20 \mathrm{~h}$, and $30 \mathrm{~h}$ of culture. The nHAAg-CG3.0 exhibited restrained growth of S. aureus as compared to nHA-Ag-CG1.5 at $30 \mathrm{~h}$ because of the presence of higher concentration of biocompatible $\mathrm{CG}$.

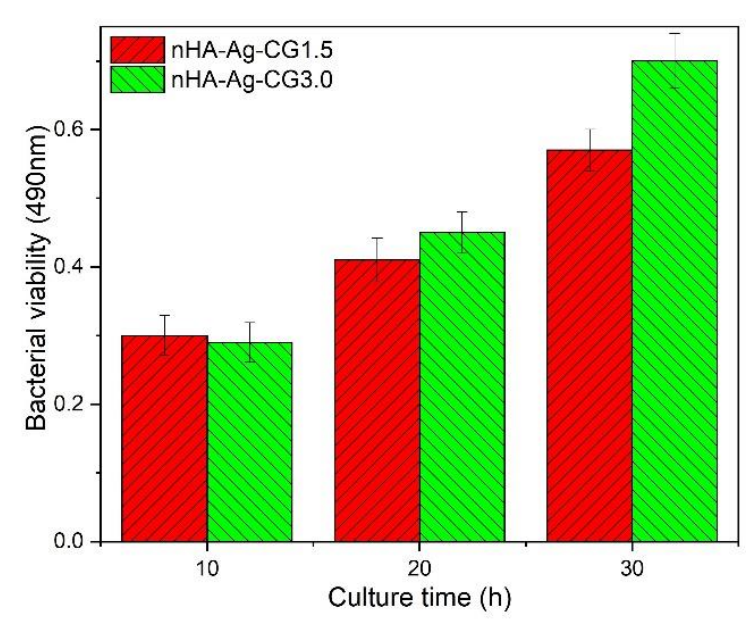

Fig. 4. Bacterial viability on the specimens

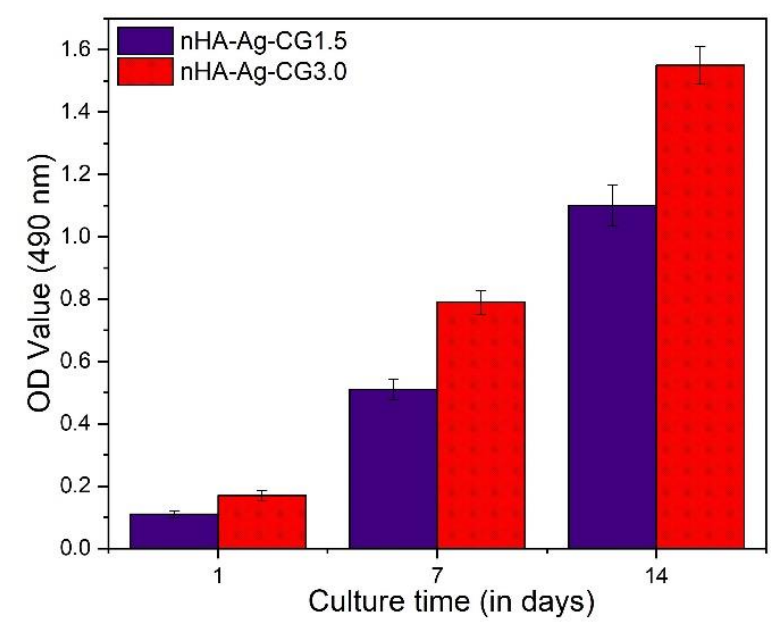

Fig. 5. MG63 cell viability on the specimens

\subsection{Cell viability assay}

The osteoblast cell viability of both the as-formed hydrogels was performed using CCK-8 assay as depicted in Fig. 5. It can be seen that higher MG63 cells were survived on the nHA-AgCG3.0. This may be attributed to the presence of higher amount of CG in the hydrogels. It has been established that $\mathrm{CG}$ is responsible for higher $\mathrm{Ca}$ deposition because of its affinity to bind $\mathrm{Ca}$ that subsequently accelerate cell differentiation giving rise to new bone formation [17].

Hence, nHA-Ag-CG3.0 may be considered to have more cytocompatibility than nHA-Ag-CG1.5. Although the cytocompatibility of the hydrogels is compromised in the presence of antibacterial agent, presence of cytocompatible HA and CG compensate the cytotoxic behavior of Ag. These hydrogels are suggestive of being used as successful scaffold materials for hosting bone tissues.

The qualitative analysis of MG63 cell attachment on the hydrogels was performed using CLSM imaging after $24 \mathrm{~h}$ of culture. DAPI staining was used to visualize nucleus of adherent cells on the hydrogels. It can be found that cell spreading and proliferation were higher in $\mathrm{nHA}-\mathrm{Ag}-$ CG3.0 hydrogels after $12 \mathrm{~h}$ of culture. This may be attributed to the higher cytocompatibility of 
nHA-Ag-CG3.0. Hence, the as-formed hydrogels may be useful in contributing to osteogenicity in the presence of antibacterial agents solving a long standing problem of dual action.

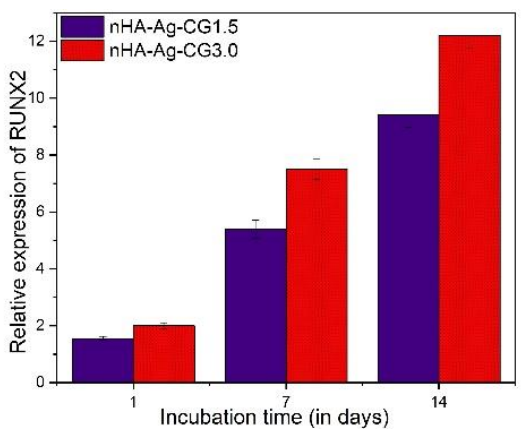

Fig. 6. Relative expression of RUNX2

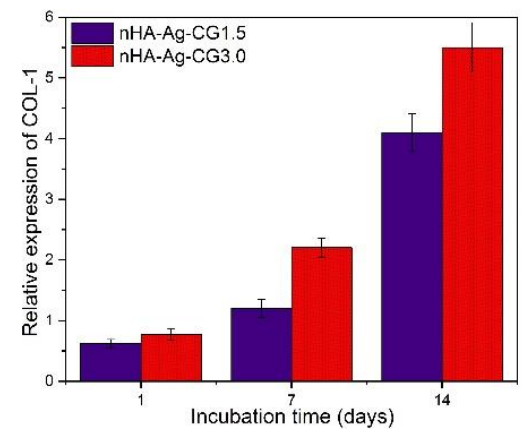

Fig. 7. Relative expression of COL-1

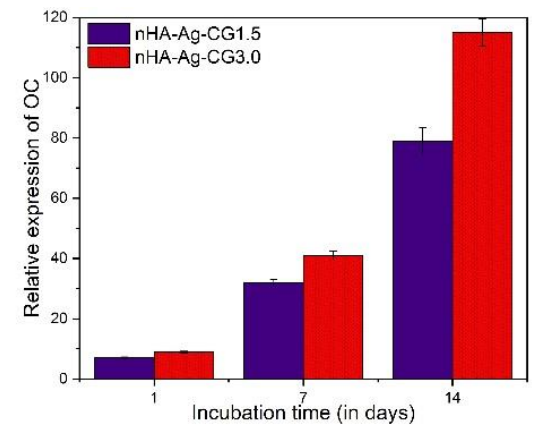

Fig. 8. Relative expression of $\mathrm{OC}$
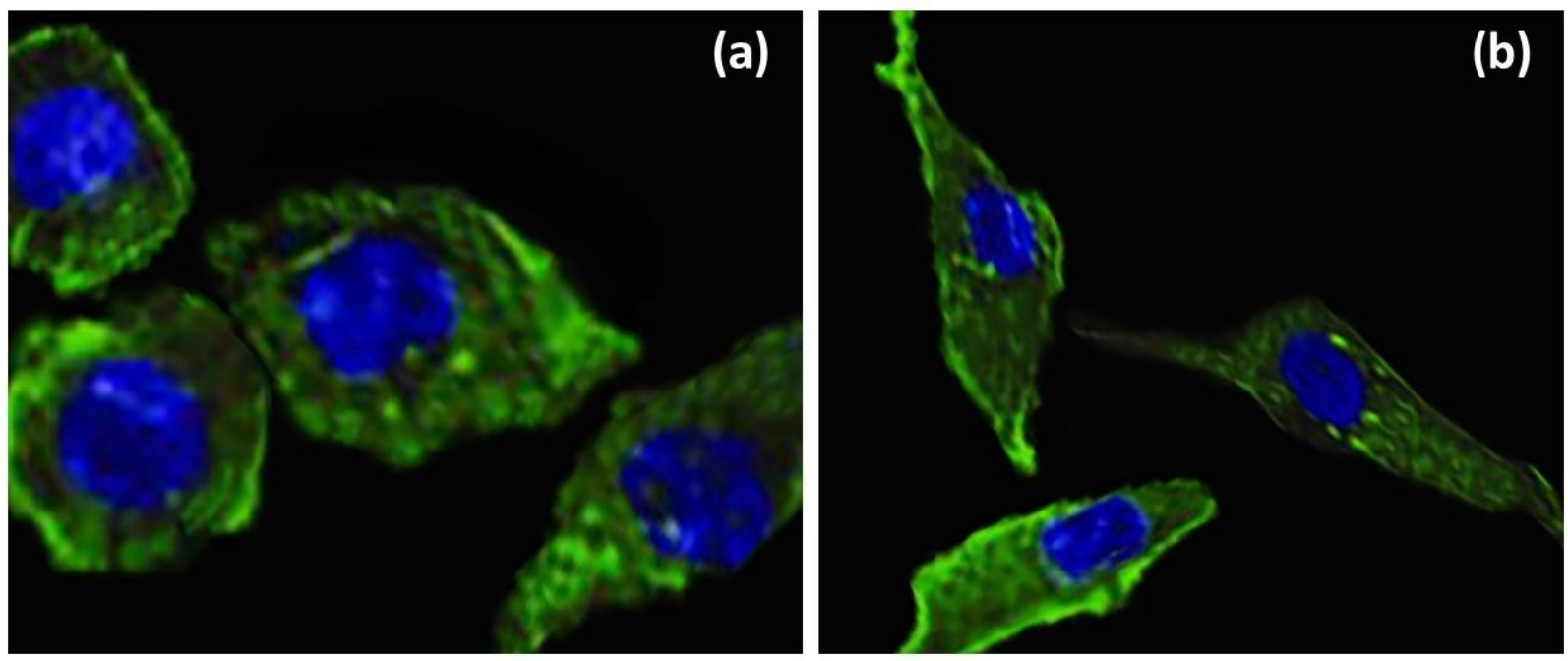

Fig. 9. CLSM images of (a) nHA-Ag-CG1.5 and (b) nHA-Ag-CG3.0

Osteogenic gene expression studies demonstrated that RUNX2 (Fig. 6) and OC (Fig. 7) expressions for nHA-Ag-CG3.0 were higher by $29 \%$ and $27 \%$ respectively as compared to nHAAg-CG1.5 while there was $40 \%$ increase in COL1 (Fig. 8) expression on day 1. On the other hand, RUNX2 and OC expressions for nHA-Ag-CG3.0 were higher by $38 \%$ and $28 \%$ respectively as compared to $\mathrm{nHA}-\mathrm{Ag}-\mathrm{CG} 1.5$ while there was $45 \%$ increase in COL1 expression on day 7. Moreover, RUNX2 and OC expressions for nHA-Ag-CG3.0 were higher by $29 \%$ and $45 \%$ respectively as compared to $\mathrm{nHA}-\mathrm{Ag}-\mathrm{CG} 1.5$ while there was $36 \%$ increase in COL1 expression on day14. The steady increase in osteogenic expressions for nHA-Ag-CG3.0 specimen may be attributed to the presence of higher concentration of carageenan that contributed for higher osteogenicity. COL-I osteogenic marker is an early marker exhibiting mineralization of bone tissues while other marker RUNX2 is expressed in the later stages during 
bone mineralization while $\mathrm{OC}$ is expressed during remodeling phase [30]. nHA-Ag-CG3.0 promoted the expression of COL-I, RUNX2 and OC markers exhibiting exceptional bioactivity. However, further experiments may be conducted to explain the complete signaling pathways and the intrinsic mechanisms involved.

Increased surface area has been shown to impact more on the cells. The polar surface of nHA is largely hydrophilic similar to carageenan that helps in the adsorption of protein based on the principles of ionic and hydrogen bonding. Hydrophilic surfaces induce cellular activities and has been shown to stimulate vitronectin and fibronectin those in turn supports the growth of osteoblast cells protecting the bioactivity of adsorbed protein. It has been found from many studies that $\mathrm{Ca}^{2+}$ and $\mathrm{PO}_{4}{ }^{3-}$ of $\mathrm{HA}$ and $\mathrm{SO}_{4}{ }^{2-}$ of $\mathrm{CG}$ helps in promoting adsorption of many proteins [31].

\subsection{CLSM analysis}

CLSM analysis (Fig. 9) of the specimens at $6 \mathrm{~h}$ of culture depicted that osteoblasts adhered to the surface of specimens and offered circular morphology on the nHA-Ag-CG1.5 specimen and elongated morphology on the nHA-Ag-CG3.0 specimen at $6 \mathrm{~h}$ of culture. Higher magnification of CLSM images indicated distinct differences in the morphology of cells in both the specimens and visibility of extended F-actin cytoskeletal structure of cells on nHA-Ag-CG3.0 specimen. On the nHA-Ag-CG1.5 specimen, MG63 cells offered low cytoplasm expansion in distinctive osteoblast cells with rounded morphology with clear visibility of the nuclei. Surveillance of MG63 cells at $6 \mathrm{~h}$ of culture offered evidence of cell adhesion on the surfaces of specimens and on the cytoplasm expansion and the attainment of distinctive cell morphology, actions which are strongly reliant on the reform of the F-actin cytoskeleton. It is an important observation since the F-actin cytoskeleton takes primary role in the maintenance of the shapes and junctions of cells that provides mechanical backing to the cells.

\section{Conclusions}

The as-formed hydrogels were hydrophilic in nature. nHA-Ag-CG3.0 hydrogel improved its mechanical strength after incorporation of higher CG concentration. The Young's modulus of nHA-Ag-CG3.0 was higher than nHA-Ag-CG1.5 demonstrating better interfacial compatibility of their matrix with respect to the reinforcement. The higher swelling ratio and biodegradability of nHA-Ag-CG3.0 is envisaged to induce better cell adhesion and proliferation. The bacterial 
cell viability of $S$. aureus on $\mathrm{nHA}-\mathrm{Ag}-\mathrm{CG} 3.0$ showed to have restrained growth as compared to nHA-Ag-CG1.5. nHA-Ag-CG3.0 may be considered to have more cytocompatibility than nHAAg-CG1.5 due to higher concentration of CG. Osteogenic gene expression studies demonstrated that nHA-Ag-CG3.0 significantly elevated the expression of osteogenic genes such as COL1, RUNX2 and OC during the culture times of 1, 7 and 14 days. Incorporation of higher CG amount in the presence of $\mathrm{Ag}$ was found to be more cytocompatible with better mechanical properties to be used as an ideal scaffold material.

\section{References}

1. X. Tong and F. Yang, Recent Progress in Developing Injectable Matrices for Enhancing Cell Delivery and Tissue Regeneration. Adv Healthc Mater 7, 1701065 (2018).

2. S. Swain, T. R. Rautray, Effect of Surface Roughness on Titanium Medical Implants. In: Swain B.P. (ed.) Nanostructured Materials and their Applications. Materials Horizons: From Nature to Nanomaterials. Springer, Singapore (2021).

3. M. G. Raucci, C. Demitri, A. Soriente, I. Fasolino, A. Sannino, L. Ambrosio, Gelatin/nano-hydroxyapatite hydrogel scaffold prepared by sol-gel technology as filler to repair bone defects. J Biomed Mater Res Part A 106, 2007-2019 (2018).

4. T. R. Rautray, S. Swain, K. H. Kim, Formation of anodic TiO2 nanotubes under magnetic field, Adv Sci Lett 20, 801-803 (2014).

5. S. Yang, K. F. Leong, Z. Du, C. K. Chua, The design of scaffolds for use in tissue engineering. Part I. Traditional factors. Tissue Eng 7, 679-689 (2001).

6. B. Mohapatra, T. R. Rautray, Strontium-substituted biphasic calcium phosphate scaffold for orthopedic applications. J Korean Ceram Soc 1-9, (2020).

7. H. W. Kim, J. C. Knowles, H. E. Kim, Hydroxyapatite and gelatin composite foams processed via novel freeze-drying and crosslinking for use as temporary hard tissue scaffolds. J Biomed Mater Res 72, 136-145 (2005).

8. J. Han, Z. Zhou, R. Yin, D. Yang, J. Niw, Alginate-chitosan-hydroxyapatite polyelectrolyte complex porous scaffolds: Preparation and characterization. Int J Biol Macromol 46, 199-205 (2010).

9. T. R. Rautray, B. Mohapatra, K. H. Kim, Fabrication of strontium-hydroxyapatite scaffolds for biomedical applications. Adv Sci Lett 20, 879-881 (2014).

10. T. R. Rautray, V. Vijayan, S. Panigrahi, Synthesis of hydroxyapatite at low temperature, Ind J Phy 81, 95-98 (2007). 
11. S. Swain, R. N. Padhy, T. R. Rautray, Electrically stimulated hydroxyapatite-barium titanate composites demonstrate immunocompatibility in vitro. $J$ Korean Ceram Soc 57, 495-502 (2020).

12. S. Swain, T. R. Rautray, R. Narayanan, Sr, Mg, and Co substituted hydroxyapatite coating on $\mathrm{TiO} 2$ nanotubes formed by electrochemical methods. Adv Sci Lett 22, 482-487 (2016).

13. S. Swain, T. R. Rautray, Silver doped hydroxyapatite coatings by sacrificial anode deposition under magnetic field. J Mater Sci: Mater Med 28, 160 (2017).

14. T. R. Rautray, K. H. Kim, Synthesis of silver incorporated hydroxyapatite under magnetic field. Key Eng Mater 493, 181-185 (2012).

15. J. Liu, X. Zhan, J. Wan, Y. Wang, C. Wang, Review for carrageenan-based pharmaceutical biomaterials: favourable physical features versus adverse biological effects. Carbohydr Polym 121, 27-36 (2015).

16. V. D. Prajapati, P. M. Maheriya, G. K. Jani, H. K. Solanki, Carrageenan: a natural seaweed polysaccharide and its applications. Carbohydr Polym 105, 97-112 (2014).

17. S. Mirza, R. Jolly, I. Zia, M. S. Umar, M. Owais, M. Shakir, Bioactive Gum Arabic/אCarrageenan-Incorporated Nano-Hydroxyapatite Nanocomposites and Their Relative Biological Functionalities in Bone Tissue Engineering. ACS Omega 5, 11279-11290 (2020).

18. M. Bornhoft, M. Thommes, P. Kleinebudde, Preliminary assessment of carrageenan as excipient for extrusion/spheronization. Eur J Pharm Biopharm 59, 127-131 (2005).

19. S. Swain, R. N. Padhy, T. R. Rautray, Polarized piezoelectric bioceramic composites exhibit antibacterial activity, Mater Chem Phy 239, 122002 (2020).

20. S. Swain, T. R. Rautray, Estimation of Trace Elements, Antioxidants, and Antibacterial Agents of Regularly Consumed Indian Medicinal Plants. Biol Trace Element Res 1-9 (2020).

21. S. Swain, R. D. K. Misra, C. K. You, Tapash R Rautray, $\mathrm{TiO}_{2}$ nanotubes synthesised on Ti-6Al-4V ELI exhibits enhanced osteogenic activity: A potential next-generation material to be used as medical implants, Mater Technolo 1-7,2020

22. Y. Tu, N. Chen, C. Li , H. Liu , R. Zhu , S. Chen, Q. Xiao, J. Liu, S. Ramakrishna, L. He, Advances in injectable self-healing biomedical hydrogels. Acta Biomaterialia 90, 1-20 (2019).

23. A. A. Amini, L. S. Nair, Injectable hydrogels for bone and cartilage repair, Biomed Mater 7, 024105 (2012). 
24. J. I. G. Ocampo, N. Bassous, C. P. O. Orozco, T. J. Webster, Evaluation of cytotoxicity and antimicrobial activity of an injectable bone substitute of carrageenan and nano hydroxyapatite. J Biomed Mater Res Part A 106, 2984-2993 (2018).

25. L. F. B. Nogueira, B. C. Maniglia, D. R. T. Blácido, A. P. Ramos, Organic-inorganic collagen/iota-carrageenan/hydroxyapatite hybrid membranes are bioactive materials for bone regeneration. J Appl Polym Sci, 48004 (2019).

26. B. Maharjan, D. Kumar, G. P. Awasthi, D. P. Bhattarai, J. Y. Kim, C. H. Park, C. S. Kim, Synthesis and characterization of gold/silica hybrid nanoparticles incorporated gelatin methacrylate conductive hydrogels for $\mathrm{H} 9 \mathrm{C} 2$ cardiac cell compatibility study. Composites B 177, 107415 (2019).

27. D. A. Puleo, A. Nanci, Understanding and controlling the bone-implant interface. Biomater 20, 2311-2321 (1999).

28. I. M. E. Sherbiny, M. H. Yacoub, Hydrogel scaffolds for tissue engineering: Progress and challenges. Global Cardiolo Sci Practice 38, 317-342 (2013).

29. S. Mishra, T. R. Rautray, Silver-incorporated hydroxyapatite-albumin microspheres with bactericidal effects. J Korean Ceram Soc 57, 175-183 (2020).

30. C. Bialorucki, G. Subramanian, M. Elsaadany, In situ osteoblast mineralization mediates post-injection mechanical properties of osteoconductive material, J Mech Behav Biomed Mater 38, 143-153 (2014).

31. D. M. Rivera-Chacon, M. Alvarado-Velez, C.Y. Acevedo-Morantes, S.P. Singh, E. Gultepe, D. Nagesha, S. Sridhar, J.E. Ramirez-Vick, Fibronectin and vitronectin promote human fetal osteoblast cell attachment and proliferation on nanoporous titanium surfaces, J Biomed Nanotechnol 9, 1092-1097 (2013). 